\title{
ARTICLE
}

Received 19 Mar 2014 | Accepted 5 Jul 2014 | Published 20 Aug $2014 \quad$ DOl: 10.1038/ncomms5598 OPEN

\section{Thermoelectric Seebeck effect in oxide-based resistive switching memory}

Ming Wang ${ }^{1, \star}$, Chong $\mathrm{Bi}^{1}{ }^{1} \star$, Ling $\mathrm{Li}^{1}$, Shibing Long ${ }^{1}$, Qi Liu ${ }^{1}$, Hangbing Lv ${ }^{1}$, Nianduan Lu${ }^{1}$, Pengxiao Sun ${ }^{1}$ \& Ming Liu' ${ }^{1}$

Reversible resistive switching induced by an electric field in oxide-based resistive switching memory shows a promising application in future information storage and processing. It is believed that there are some local conductive filaments formed and ruptured in the resistive switching process. However, as a fundamental question, how electron transports in the formed conductive filament is still under debate due to the difficulty to directly characterize its physical and electrical properties. Here we investigate the intrinsic electronic transport mechanism in such conductive filament by measuring thermoelectric Seebeck effects. We show that the small-polaron hopping model can well describe the electronic transport process for all resistance states, although the corresponding temperature-dependent resistance behaviours are contrary. Moreover, at low resistance states, we observe a clear semiconductor-metal transition around $150 \mathrm{~K}$. These results provide insight in understanding resistive switching process and establish a basic framework for modelling resistive switching behaviour.

\footnotetext{
${ }^{1}$ Lab of Nanofabrication and Novel Device Integration, Institute of Microelectronics, Chinese Academy of Sciences, Beijing 100029, China. ${ }^{\star}$ These authors contributed equally to this work. Correspondence and requests for materials should be addressed to M.L. (email: liuming@ime.ac.cn).
} 
$\mathrm{R}$ eversible resistive switching (RS) in the transition metal oxide is a typical electric field- and current-induced phenomenon that the resistance $(R)$ strongly relates with the history of applied voltage and current ${ }^{1-3}$. It has attracted considerable attention for future information storage and processing, such as resistive random access memory (RRAM), reconfigurable logic circuit and artificial neuromorphic networks ${ }^{2-5}$. The switching between high-resistance state and low-resistance state (LRS) usually involves the generation, transport and recombination of defects, such as oxygen vacancies and metal ions, which causes the structure or composition modulation of the 'active' switching region, usually called conductive filament $(\mathrm{CF})^{6-9}$. Understanding the charge transport in the formed CF not only benefits for further understanding RS phenomenon, but also is critical for optimizing RS-based devices in practical applications. Owing to the limitations in spatial resolution and the sensitivity of traditional material and electrical characterization techniques, it is difficult to directly measure the basic electronic transport properties of the formed CF. Although several electronic transport mechanisms have been suggested based on the current-voltage $(I-V)$ characteristics or temperature dependence of $R$, there are many discrepancies among these proposed electronic transport mechanisms. Even for the similar device structures with the same value of LRS resistance, the proposed transport mechanisms are also quite contradictory ${ }^{9-13}$.

Seebeck effect, a typical thermoelectric phenomenon that directly converts the temperature difference to electric field ${ }^{14}$, can provide deeper insights into the energetics of dominant charge transport process, and the Seebeck voltage measurement has become a high sensitive means to image structural or electronic disorder ${ }^{15,16}$. The Seebeck coefficient ${ }^{17,18}$,

$$
S=\frac{\int\left(E-E_{\mathrm{F}}\right) \sigma(E) d E}{q T \int \sigma(E) d E}
$$

reflects the asymmetry of $\sigma(E)$ with respect to $E_{\mathrm{F}}$, where $\sigma(E)$ is the energy-dependent electrical conductivity distribution function, $q$ is the basic charge of the carrier, $E_{\mathrm{F}}$ is the Fermi energy and $T$ is the absolute temperature. This asymmetry of $\sigma(E)$ can result from energy-dependent carrier scattering, defect energy level around $E_{\mathrm{F}}$ or the asymmetry of the electronic density of states. Therefore, $S$ can reflect the basic charge transport mode, such as the band-like transport or the variable-range hopping transport ${ }^{18,19}$. In addition, in contrary to $I-V$ measurements, the Seebeck voltage does not depend on the number of conductive paths and interfacial contact ${ }^{14,20}$ (see Supplementary Fig. 1 and Supplementary Note 1), and thus reveals the intrinsic electronic transport properties.

In the present study, we choose the $\mathrm{Ti} / \mathrm{HfO}_{x} / \mathrm{Pt} \mathrm{RS}$ structure as a typical example to investigate the electronic transport mechanism in metal oxide-based RRAM by measuring the Seebeck effect. The experimental results show that the measured $S$ of $\mathrm{Ti} / \mathrm{HfO}_{x} / \mathrm{Pt}$ sample almost keeps constant at $-80 \mu \mathrm{VK}^{-1}$ when $78 \Omega<R<10^{4} \Omega$, and then sharply decreases to about $-160 \mu \mathrm{V} \mathrm{K}^{-1}$ for $R \sim 10^{6} \Omega$ at room temperature. The negative $S$ indicates $n$-type electrical transport. The temperature dependence of $S$ between 150 and $300 \mathrm{~K}$ demonstrates the intrinsic electrical transport mechanism is the same for all different resistance states, although the corresponding $R$ versus temperature presents different characteristics. We show that all $S$ characteristics under different temperatures and resistances can be well explained by small-polaron hopping model and suggest that the small-polaron hopping process between vacancies dominates the main electron transport in CF. Moreover, a clear semiconductor-metal transition (SMT) is also observed for $R<\sim 106 \Omega$ when the temperature is below $150 \mathrm{~K}$.

\section{Results}

Sample configuration and $\mathrm{RS}$ characteristics. The typical W 40/Ti $10 / \mathrm{HfO}_{x} 8 / \mathrm{Pt} 60 / \mathrm{Ti} 5$ (nm) RRAM structures are used in this experiment. The bottom and top Pt 30/Ti $5(\mathrm{~nm})$ heating layers and two 70-nm-thick $\mathrm{SiO}_{2}$ isolation layers are, respectively, deposited before and after depositing $\mathrm{W} / \mathrm{Ti} / \mathrm{HfO}_{x} / \mathrm{Pt} / \mathrm{Ti}$ structure. The schematic sample structure and experimental setup are shown in Fig. 1a, where $I_{\mathrm{b}}$ and $I_{\mathrm{t}}$ are used to heat bottom and top surfaces of RRAM structure, and a Keithley sourcemeter 2440 and a voltmeter are used to operate RRAM and measure the Seebeck effect, respectively. The temperature gradient is created by Joule heating bottom and top Pt/Ti layers, and their resistances are used to evaluate the established temperature gradient (see Methods and Supplementary Fig. 2). It should be mentioned that the influence of heating current on RRAM operations can be completely suppressed by two $70-\mathrm{nm} \mathrm{SiO}_{2}$ isolation layers. We first preform the usual RRAM measurement ${ }^{2}$, forming, set and reset process, to confirm that our $\mathrm{W} / \mathrm{Ti} / \mathrm{HfO}_{x} / \mathrm{Pt} / \mathrm{Ti}$ devices show reliable RS behaviours, as shown in Fig. 1b. After the initial forming process, where voltage is swept from 0 to $+4 \mathrm{~V}$ with the compliance current $I_{\mathrm{cc}}$ of $1 \mathrm{~mA}$, the stable hysteresis-like $I-V$ curves between $-1.5 \mathrm{~V}$ and $2 \mathrm{~V}$ during the subsequent reset and set processes are achieved, indicating that the sample works well as a RRAM.

Seebeck measurement. The Seebeck coefficient at a given temperature is determined by linearly fitting $\Delta V$ versus $\Delta T$ by using $\Delta V=-S \Delta T^{21}$, where $\Delta V$ is the measured Seebeck voltage and $\Delta T$ is the temperature difference across the $\mathrm{HfO}_{x}$ layer. Before measuring the Seebeck voltage, the sample resistance is first set to a fixed resistance state. Figure $1 \mathrm{c}$ shows the typical measured Seebeck voltage as a function of $I_{\mathrm{t}}$ for $R=670 \Omega$ when $I_{\mathrm{b}}=0 \mathrm{~mA}$ at $300 \mathrm{~K}$. It is clearly shown that the measured Seebeck voltage increases with current and does not depend on the current flow direction. The positive Seebeck voltage at the hot end indicates the electrons are the main carriers ${ }^{14}$. The inset of Fig. 1c shows the corresponding voltage as a function of $I_{\mathrm{t}}^{2}$. As $\Delta T$ is proportional to $I_{\mathrm{t}}^{2}$ (see Supplementary Fig. 2c), the linear dependence of $\Delta V$ on $I_{\mathrm{t}}^{2}$ indicates that the measured voltage is proportional to the temperature difference at $\mathrm{HfO}_{x}$ layer, which is the typical Seebeck voltage characteristics. When sweeping $I_{\mathrm{b}}$ under $I_{\mathrm{t}}=0 \mathrm{~mA}$, an identical tendency of measured voltage as a function of applied $I_{\mathrm{b}}$ is also observed, except that the sign is negative. When $I_{\mathrm{t}}=60 \mathrm{~mA}$ and $I_{\mathrm{b}}=0 \mathrm{~mA}, \Delta T$ is $\sim 1.3 \mathrm{~K}$, which corresponds to an $S$-value of $\sim 84 \mu \mathrm{V} \mathrm{K}^{-1}$ for $R=670 \Omega$ at $300 \mathrm{~K}$. Such large $S$-value of several tens of $\mu \mathrm{VK}^{-1}$, which is much larger than that for typical metal ${ }^{14}$, indicates that the electron transport cannot be understood from the metallic band-like electrical transport model. Figure 1d shows the measured Seebeck voltage as a function of $I_{\mathrm{t}}$ at several representative resistance states. For the LRS of $R<10^{4} \Omega$, the Seebeck coefficient is almost identical for different resistance states; however, for $R>10^{4} \Omega$, the value of $S$ extracted from linear fitting of $\Delta V$ versus $\Delta T$ increases with $R$ and can reach up to $160 \mu \mathrm{VK}^{-1}$ for the $R$ of $\sim 10^{6} \Omega$. For $R>10^{6} \Omega$ or virgin sample without any RS operation, no Seebeck signal is observed.

To offer the further insight into the electronic transport in $\mathrm{CF}$, we measure $S$ at different temperatures. Here we still use the sample stage temperature as $T$ due to the small $\Delta T$ compared with sample stage temperature. Figure 2a shows the temperature dependence of $S$ between 150 and $300 \mathrm{~K}$ at several representative resistance states. The corresponding $R$ versus $T$ is also simultaneously measured as shown in Fig. $2 \mathrm{~b}$. For $R=8.4 \mathrm{k} \Omega$, $R$ decreases with increasing $T$, which corresponds to a conventional semiconductor behaviour, whereas for LRS of $R=78 \Omega$ $R$ increases with increasing $\mathrm{T}$, which is the typical metallic 

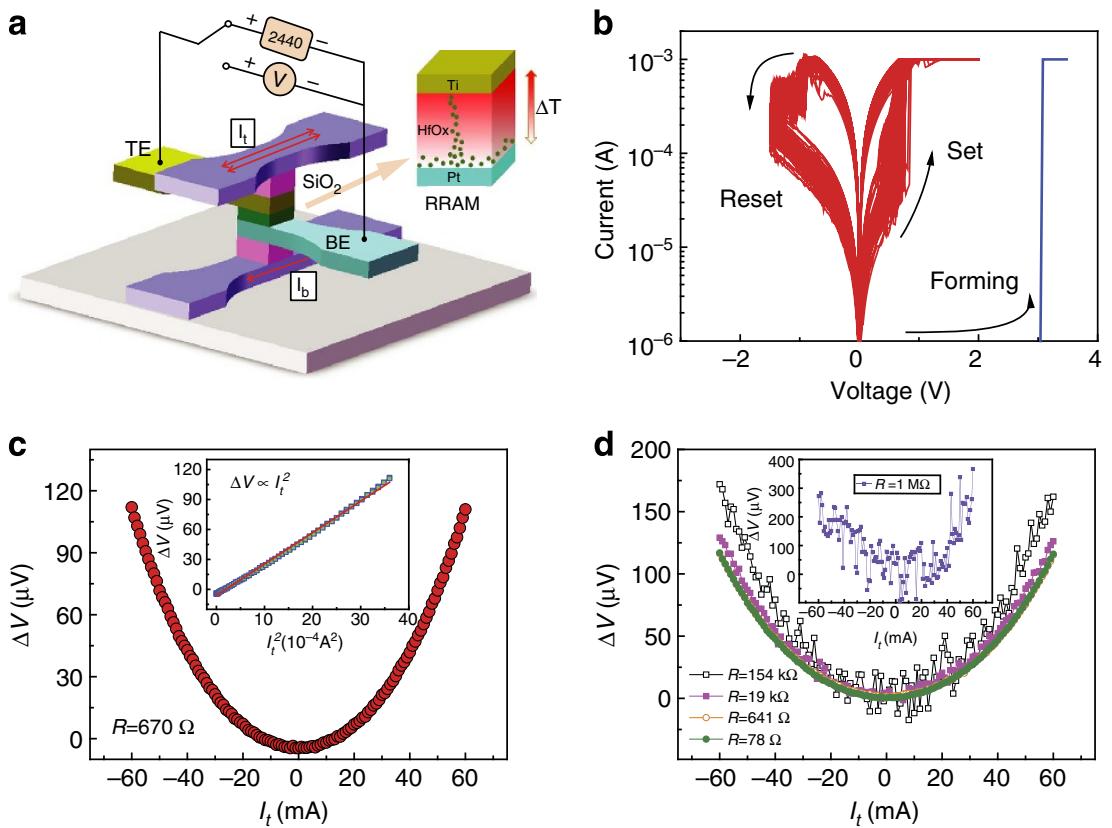

Figure 1 | Measurement of the Seebeck voltage in oxide-based RRAM at $\mathbf{3 0 0}$ K. (a) Schematic sample structure and corresponding the Seebeck measurement setup. $I_{\mathrm{t}}$ and $l_{\mathrm{b}}$ are the heating currents for top and bottom surfaces, respectively. Keithley sourcemeter 2440 is used to operate RRAM and the voltmeter is used to measure the Seebeck voltage. (b) $I-V$ characteristics of $\mathrm{Ti} / \mathrm{HfO}_{x} / \mathrm{Pt}$ structure in forming, set and reset process. The initial forming process is done by sweeping voltage from 0 to $+4 \mathrm{~V}$ with the compliance current $I_{c c}$ of $1 \mathrm{~mA}$, and the subsequent reset and set processes are performed by sweeping voltage between -1.5 and $2 \mathrm{~V}$ with $I_{\mathrm{cc}}$ of 100 and $1 \mathrm{~mA}$, respectively. (c) Measured Seebeck voltage as a function of $I_{\mathrm{t}}$ when $I_{\mathrm{b}}=0 \mathrm{~mA}$ for $R=670 \Omega$. The inset shows the linear fitting (solid line) of the Seebeck voltage (symbols) versus $I_{\mathrm{t}}^{2}$. (d) Measured Seebeck voltages at several representative resistance states from $78 \Omega$ to $1 \mathrm{M} \Omega$ (inset).

behaviour. Surprisingly, as shown in Fig. 2a, $S$ for all the resistance states show the identical tendency of temperature dependence. As we mentioned above, the Seebeck voltage does not depend on the interfacial contact and only reflects the basic electronic properties of CFs; thus, the identical tendency of $S$ versus $T$ indicates all the resistance states have the same intrinsic electrical transport mechanism.

As we mentioned above, because the Seebeck voltage does not depend on the interfacial contact and the electrical measurement can be strongly influenced by contact, especially for the LRS, it is reasonable to assume that the different $R$ versus $T$ tendencies at different resistance states are due to interfacial contact resistance. To confirm this assumption, we investigate the contact resistance for the samples with different $\mathrm{HfO}_{x}$ thickness (see Supplementary Fig. 3 and Supplementary Note 2). Before measuring the contact resistance, the sample is intentionally broken down to minimize the possible CF contribution. A linear relationship between the resistance of broken-down sample and the $\mathrm{HfO}_{x}$ thickness with the intercept of $9.7 \Omega$ is observed (see Supplementary Fig. 3a), which indicates that the contact resistance almost keeps constant at $\sim 10 \Omega$ for the sample with different $\mathrm{HfO}_{x}$ thickness. On the other hand, the temperature dependence of the contact resistance presents a metallic behaviour (see Supplementary Fig. 3b). Therefore, for $R=8.4 \mathrm{k} \Omega$ case, where the contribution of contact resistance is much smaller than that for $R=78 \Omega$, the decreasing tendency of $R$ with increasing temperature reveals the intrinsic electrical transport mechanism in CF. Because of the same electrical transport mechanism resulted from $S$ versus $T$, we believe the metallic increasing tendency of $R$ with increasing temperature for $R=78 \Omega$ is due to the contribution of contact resistance, which results in the metallic behaviour as mentioned above. It should be mentioned that although the resistance of $\mathrm{CF}$ is still larger than the contact resistance for $R=78 \Omega$, the resistance change of contact is larger than that for CF when $T$ increases from 150 to $300 \mathrm{~K}$. Therefore, the temperaturedependent behaviour of the contact resistance dominates the total measured resistance behaviour when $R=78 \Omega$, leading to a metallic behaviour as shown in Fig. 2b. As we discussed below, the decreasing tendency of $R$ with increasing temperature is the typical semiconductor transport behaviour, which can be understood by the small-polaron hopping transport model.

Seebeck coefficient and resistance below $150 \mathrm{~K}$. Another remarkable result is the temperature dependences of $S$ for $R=8.4 \mathrm{k} \Omega$ and $R=78 \Omega$ are quite different at lower-temperature region below $150 \mathrm{~K}$. As shown in Fig. 3a, in contrary to a continuous decrease tendency of the $8.4 \mathrm{k} \Omega, S$ of $78 \Omega$ tends to be saturated when $T<150 \mathrm{~K}$. We attribute the saturated $S$ at lower temperature to the possible SMT. First, in our case, the constant value of $S$ about several $\mu \mathrm{V} \mathrm{K}^{-1}$ below $150 \mathrm{~K}$, which does not depend on the temperature anymore, is the typical metallic electrical transport behaviours ${ }^{14}$. Second, to further identify temperature-induced SMT, we measured temperature dependence of $R$ for different resistance states from $64 \Omega$ to $8.4 \mathrm{k} \Omega$ under the temperature from 10 to $300 \mathrm{~K}$, as shown in Fig. 3b. For clarity, the contact resistance has been subtracted from every resistance value (see Supplementary Fig. 3b). For LRS of 82 and $64 \Omega$, two distinct regions with the opposite temperature dependences of $R$ are observed, and the transition temperature between two regions decreases with increasing $R$. For $R>106 \Omega$, the transition temperature becomes close to $0 \mathrm{~K}$, and no clear SMT is observed. We speculate the observed SMT may be due to the temperature-induced Fermi level movement ${ }^{22}$, and more details need to be further clarified by theory.

\section{Discussion}

We will show below the temperature dependence of $S$ can be quantitatively explained by the small-polaron hopping transport 

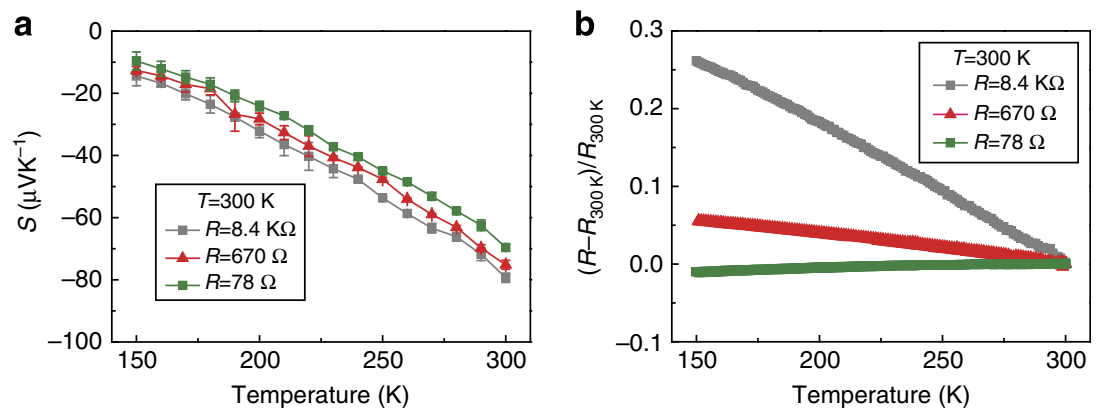

Figure 2 | Temperature-dependent measurement results. (a) Seebeck coefficient as a function of temperature for several representative resistance states $(R=78 \Omega, 670 \Omega$ and $8.4 \mathrm{k} \Omega)$ in oxide-based RRAM. (b) Temperature dependence of normalized resistance by the resistance at $300 \mathrm{~K}\left(R_{300 \mathrm{~K}}\right)$.
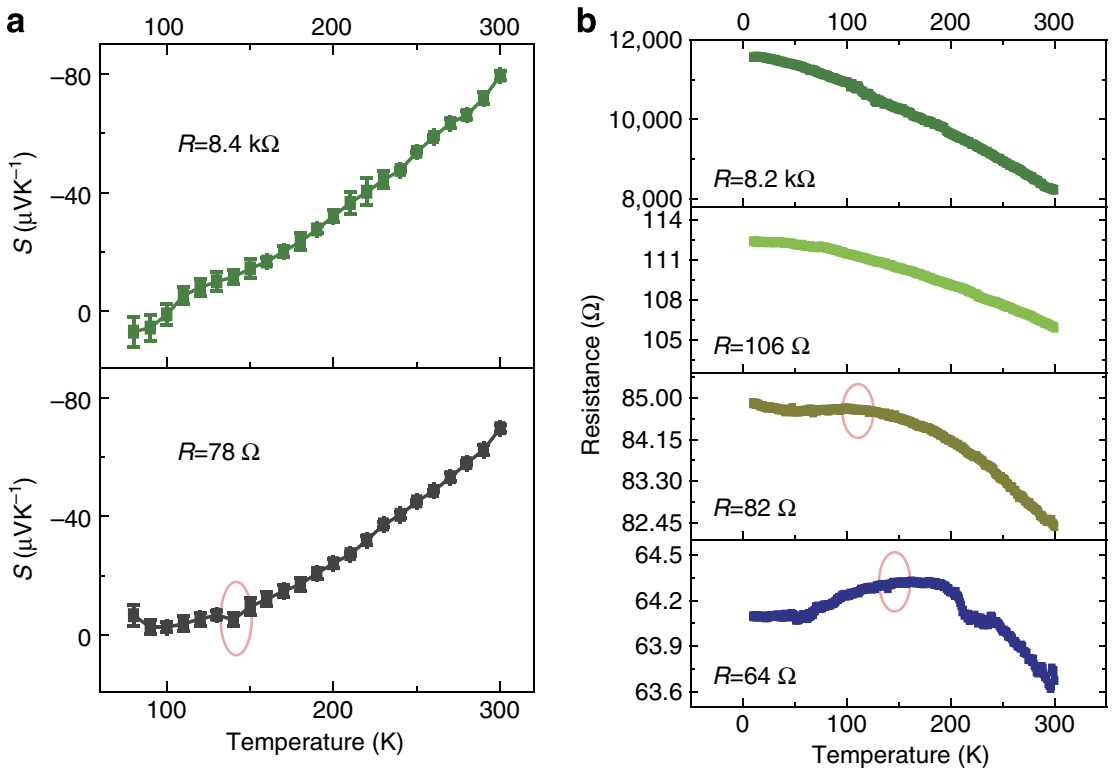

Figure 3 | Measured SMT around 150 K. (a) Representative temperature dependence of the Seebeck coefficient when $R=78 \Omega$ and $8.4 \mathrm{k} \Omega$ between 78 and $300 \mathrm{~K}$. (b) Temperature dependence of resistance from 10 to $300 \mathrm{~K}$ for several representative resistance states. The contact resistance has been deducted. The red circles represent the transition temperature regions.

model. Based on this model, the Seebeck coefficient now can be simplified to the sum of two terms ${ }^{23}$ (see Supplementary Note 3)

$$
S=\mathrm{A}+\mathrm{B} T \text {. }
$$

The first term $A$ is the standard term, which is proportional to the average change of the entropy. The second term $\mathrm{B}=k z J^{2} / 2 q E_{\mathrm{b}}^{3}$ is proportional to the transported average lattice vibrational energy associated with charge hopping, where $k$ is Boltzmann constant, $J$ is the intersite transfer energy, $E_{\mathrm{b}}$ is the small-polaron binding energy and $z$ is the number of nearest neighbours. Figure 4 shows the fitting results of Fig. 2a by equation (2) using parameters $A=58,63,68$ and $B=-0.445,-0.451,-0.456$ for $R=8.4 \mathrm{k} \Omega$, $670 \Omega, 78 \Omega$, respectively. With decreasing the resistance, which is corresponding to the increasing of the oxygen vacancy concentration, the entropy contribution $A$ increases. This occurs because of the addition of the active oxygen vacancy due to the increasing fraction of the filled unfavourable oxygen vacancy sites in energy as the small-polaron electron band broadens. Therefore, the average energy disparity between oxygen vacancy sites increases with the addition of oxygen vacancy. Otherwise, with the increasing of oxygen vacancy, the mean energy separation between oxygen vacancy decreases; as a result,

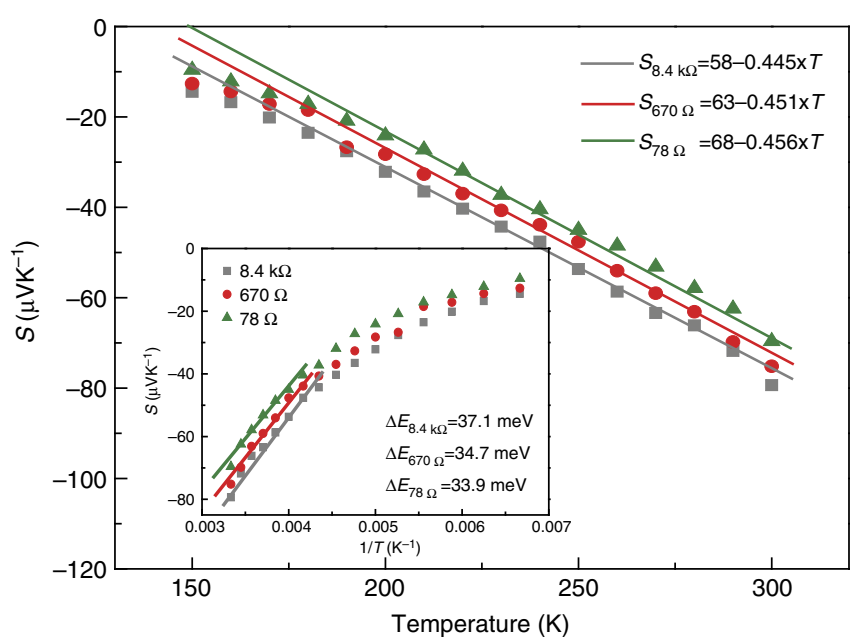

Figure 4 | Fitting results of temperature-dependent Seebeck coefficient. The fitting results of the Seebeck coefficient $S$ versus $T$ for several representative resistance states $(R=78 \Omega, 670 \Omega$ and $8.4 \mathrm{k} \Omega$ ). The inset plots $S$ versus $1 / T$ and the corresponding fitting results. 
a

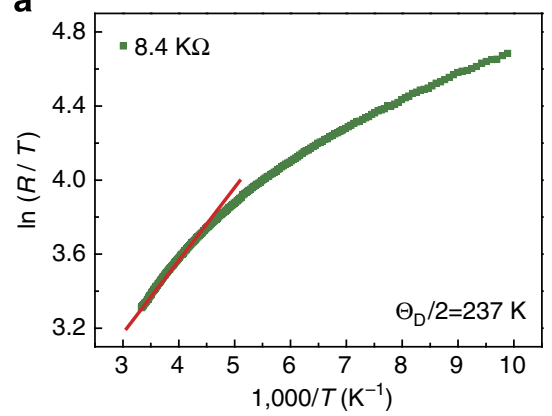

b

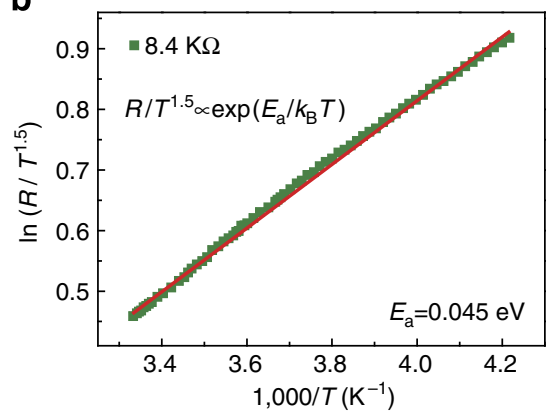

Figure 5 | Fitting results of temperature-dependent resistance. (a) Inverse temperature dependence of $\ln (R / T)$ in high-temperature range. The temperature where the slope changes from linearity is $\theta_{\mathrm{D}} / 2$. (b) The fitting results of $\ln \left(R / T^{1.5}\right)$ versus $(1,000 / T)$ above $\theta_{\mathrm{D}} / 2(237 \mathrm{~K})$ by using small polaron-hopping model. The slope gives the activation energy from electrical measurement.

the characteristic transfer energy for the intervacancy hopping increases, and thus the resultant $B$ increases with oxygen vacancy concentration. In addition, by fitting $S$ versus $1 / T$ using the more general Mott Seebeck model ${ }^{24}$, the activation energy of electron hopping between oxygen vacancies $\Delta E=37.1,34.7$ and $33.9 \mathrm{meV}$ for $R=8.4 \mathrm{k} \Omega, 670 \Omega$ and $78 \Omega$, respectively (see the inset of Fig. 4).

Furthermore, to minimize the effect of contact resistance, we take $R=8.4 \mathrm{k} \Omega$ as an example to extract the charge transport parameters by using the small-polaron hopping model. First, we evaluate the Debye temperature by fitting the measured resistance in high-temperature range. Figure $5 \mathrm{a}$ shows the fitting results by plotting $\ln (R / T)$ versus $1 / T$. From this plot, the half Debye temperature, $\theta_{\mathrm{D}} / 2=237 \mathrm{~K}$, where the slope changes from linearity is determined. Second, according to the small-polaron hopping model (non-adiabatic) ${ }^{25}$, the expression for resistance takes the form

$$
R / T^{1.5} \infty \exp \left(\frac{E_{\mathrm{a}}}{k_{\mathrm{B}} T}\right)
$$

where $E_{\mathrm{a}}$ is the activation energy. Therefore, the activation energy can be further obtained by fitting the measured resistance using equation (3). The corresponding fitting results by using $E_{\mathrm{a}}=0.045 \mathrm{eV}$ is shown in Fig. $5 \mathrm{~b}$. The difference of the activation energy between here and the one obtained from fitting the Seebeck coefficient is due to the polaron-hopping energy ${ }^{26,27}$. Based on the value extracted here, the evaluated value for small-polaron coupling $\gamma_{\mathrm{p}}=E_{\mathrm{a}} / k_{\mathrm{B}} \theta_{\mathrm{D}} \approx 1.1$. From the value of $\gamma_{\mathrm{p}}$, an estimation of the polaron effective mass, $m_{\mathrm{p}}$, can be obtained by using $m_{\mathrm{p}}=m^{\star} \exp \left(\gamma_{\mathrm{p}}\right)$ with $m^{*}$ denoting the rigid lattice effective mass. The calculated values of $\gamma_{\mathrm{p}}$ are found to be smaller than 4 , indicating the weak electron-phonon interaction here $^{28}$. These results further confirm that the charge transport is dominated by the hopping of small-polaron between vacancies.

Finally, by combining the electrical and the Seebeck measurement results, the carrier concentration of $\sim 10^{19} \mathrm{~cm}^{-3}$ is estimated for $R=8.4 \mathrm{k} \Omega$ (see Methods), which is corresponding to the oxygen vacancy density within the CF. More remarkably, although the carrier concentration in lower resistance state cannot be accurately estimated due to the involvement of contact resistances, the relative carrier concentration could be approximately estimated from the overall $R$-values. The carrier concentration for $R=78 \Omega$ will be $\sim 107$ times higher than that in $R=8.4 \mathrm{k} \Omega$, and the carrier concentration $\left(\sim 10^{2 \mathrm{P}} \mathrm{cm}^{-3}\right)$ is still much less than that in epitaxial $\mathrm{HfO}_{x}{ }^{29}$. These results indicate that the oxygen vacancy density is still below the highest defect concentration and the electron transport in $\mathrm{CF}$ is dominated by hopping process.
In summary, we have measured the thermoelectric Seebeck effect in metal oxide-based RRAM and investigated the fundamental electronic transport properties of formed CF in RS process. We show, regardless of the resistance of RRAM, the charge transport in formed $\mathrm{CF}$ can be qualitatively described within the model of small-polaron hopping between oxygen vacancy sites. Moreover, a temperature-dependent SMT is observed for LRS of $R<106 \Omega$ with the transition temperature decreasing with increasing $R$. The thermoelectric Seebeck measurement, which promises to eliminate the possible contact contribution, opens a new avenue in investigating the electronic transport in RRAM-like devices.

\section{Methods}

Device fabrication. The RRAM devices with the dimension of $15 \times 15 \mu \mathrm{m}$ were obtained from $\mathrm{Pt} 30 / \mathrm{Ti} 5 / \mathrm{SiO}_{2} 70 / \mathrm{W} 40 / \mathrm{Ti} 10 / \mathrm{HfO}_{x} 8 / \mathrm{Pt} 60 / \mathrm{Ti} 5 / \mathrm{SiO}_{2} 70 / \mathrm{Pt} 30 / \mathrm{Ti}$ $5 / \mathrm{SiO}_{2} / \mathrm{Si}(\mathrm{nm})$ structures by standard nanofabrication process. Two side $\mathrm{Pt} 30 / \mathrm{Ti}$ $5(\mathrm{~nm})$ layers separated by $70 \mathrm{~nm} \mathrm{SiO}_{2}$ layers from W 40/Ti $10 / \mathrm{HfO}_{x} 8 / \mathrm{Pt} 60 / \mathrm{Ti}$ $5(\mathrm{~nm})$ are used to heat the top and bottom surfaces of RRAM. The $\mathrm{HfO}_{x}$ layer was deposited by atomic layer deposition and the other layers were deposited by sputtering. The schematic structure of the sample is shown in Fig. 1a, and other $\mathrm{SiO}_{2}$ filling layers were also deposited around the device in nanofabrication process. The top and bottom heating layers out of the RRAM region were patterned with the width sharply increasing from 15 up to $200 \mu \mathrm{m}$ to minimize the corresponding resistance contribution when evaluating temperature gradient.

Seebeck effect measurements. Before the Seebeck effect measurement, the different resistance states were achieved by ingeniously controlling the $I_{\mathrm{CC}}$ in set process or the applied voltage in reset process, respectively. The operation voltage was supplied by a Keithley 2440 sourcemeter. Next, another two Keithley 2440 sourcemeters were used to provide the Joule heating current $I_{\mathrm{b}}$ and $I_{\mathrm{t}}$, and an Agilent $34411 \mathrm{~A}$ voltmeter was used to measure the Seebeck voltage. The temperature dependence of the Seebeck coefficient was performed in a cryogenic probe station (Lakeshore, CRX-4K) with the temperature range of 78-400 K. The Seebeck voltage was also measured by sweeping $I_{\mathrm{t}}$ at each temperature point after the temperature was stabilized. The corresponding $R$ versus $T$, especially below $78 \mathrm{~K}$, was also measured by physical property measurement system (Quantum Design).

Calibration of temperature gradient across $\mathbf{H f O}_{\mathbf{x}}$ layer. We first measured the resistances of top $\left(R_{\mathrm{t}}\right)$ and bottom $\left(R_{\mathrm{b}}\right)$ heating layer as a function of temperature, and found both the resistances of top and bottom heating layer could be well fitted by a linear function. As an example, we present the typical $R_{\mathrm{t}}$ versus $T$ and the corresponding linear fitting results (see Supplementary Fig. 2a). By using the linear fitting results, we then could determine the temperature difference between top and bottom surfaces of our sample. We measured $R_{\mathrm{t}}$ as a function of top heating current $\left(I_{\mathrm{t}}\right)$, and found that $R_{\mathrm{t}}$ versus $I_{\mathrm{t}}^{2}$ could be well fitted by a linear function (see Supplementary Fig. 2b). The well linear fitting results indicate the top surface temperature $\left(T_{\mathrm{t}}\right)$ is proportional to $I_{\mathrm{t}}^{2}$. Similarly, by simultaneously measuring $R_{\mathrm{b}}$ when sweeping $I_{\mathrm{t}}$, we could also determine the linear dependence of bottom surface temperature $\left(T_{\mathrm{b}}\right)$ on $I_{\mathrm{t}}^{2}$. Therefore, the temperature difference between top and bottom surface $\left(T_{\mathrm{t}}-T_{\mathrm{b}}\right)$ as a function of $I_{\mathrm{t}}^{2}$ were obtained. For simplicity, we ignore the temperature difference across metal layers, because the thermal conductivities of these layers are much larger than those for insulating $\mathrm{SiO}_{2}$ and $\mathrm{HfO}_{x}$ layers. Therefore, the temperature difference across $\mathrm{HfO}_{x}$ layer $\left(\Delta T_{\mathrm{HfO}_{x}}\right)$ and each $\mathrm{SiO}_{2}$ 
layer $\left(\Delta T_{\mathrm{SiO}_{2}}\right)$ can be given by

$$
\frac{\Delta T_{\mathrm{HfO}_{x}}}{\Delta T_{\mathrm{SiO}_{2}}}=\frac{t_{\mathrm{HfO}_{x}}}{\kappa_{\mathrm{HfO}_{x}}} \cdot \frac{\kappa_{\mathrm{SiO}_{2}}}{t_{\mathrm{SiO}_{2}}}
$$

and

$$
\Delta T_{\mathrm{HfO}_{x}}+2 \Delta T_{\mathrm{SiO}_{2}}=T_{t}-T_{b}
$$

where $t_{\mathrm{HfO}_{x}}, t_{\mathrm{SiO}_{2}}$ is respectively the thickness of $\mathrm{HfO}_{x}$ and $\mathrm{SiO}_{2}$ layer, and $\kappa_{\mathrm{HfO}_{x}}$, $\kappa_{\mathrm{SiO}_{2}}$ is respectively the thermal conductivity of $\mathrm{HfO}_{x}$ and $\mathrm{SiO}_{2}$ layer. As the relative thermal conductivity change between the $\mathrm{HfO}_{x}$ and $\mathrm{SiO}_{2}$ layers is $<5 \%$ within the range of $80-300 \mathrm{~K}^{30,31}$, we use the relative thermal conductivity ratio $\left(\kappa_{\mathrm{HfO}_{x}}=0.005 \mathrm{Wcm}^{-1} \mathrm{~K}^{-1}\right.$ and $\left.\kappa_{\mathrm{SiO}_{2}}=0.013 \mathrm{Wcm}^{-1} \mathrm{~K}^{-1}\right)$ at $300 \mathrm{~K}$ to calculate the temperature distribution in our sample. According to equations (4) and (5), we can get the temperature difference across $\mathrm{HfO}_{x}$ layer, which shows a linear dependence on $I_{\mathrm{t}}^{2}$, and is insensitive with ambient temperature (see Supplementary Fig. $2 \mathrm{c}$ ).

Calculation of oxygen vacancy concentration. According to the activation energy equation proposed by Austin and $\mathrm{Mott}^{28}, E_{\mathrm{a}}=W_{\mathrm{H}}+W_{\mathrm{D}} / 2$, when $T>\theta_{\mathrm{D}} / 2$, where $W_{\mathrm{H}}=E_{\mathrm{a}}-\Delta E$ is the polaron-hopping energy ${ }^{27}, W_{\mathrm{D}}$ is the disorder energy. By using $E_{\mathrm{a}}=0.045 \mathrm{eV}$ and $\Delta E=0.037 \mathrm{eV}$, we can get $W_{\mathrm{H}}=0.008 \mathrm{eV}$ and $W_{\mathrm{D}}=0.074 \mathrm{eV}$. Based on the Millar-Abraham theory ${ }^{32}, W_{\mathrm{D}}=0.3 e^{2} / \varepsilon_{\mathrm{s}} R_{\mathrm{O}}$, where $\varepsilon_{\mathrm{s}}$ is the static dielectric constant and $R_{\mathrm{O}}$ is the average spacing between oxygen vacancies. If we chose $\varepsilon_{\mathrm{S}}=21$ for $\mathrm{HfO}_{x}$ film ${ }^{33}$, the $R_{\mathrm{O}}$ will be $3.5 \mathrm{~nm}$, and the oxygen vacancy concentration $n$ is $\sim 10^{19} \mathrm{~cm}^{-3}$ for $R=8.4 \mathrm{k} \Omega$. Furthermore, we can estimate $n \sim 10^{21} \mathrm{~cm}^{-3}$ for $R=78 \Omega$. For both cases, the oxygen vacancy concentration is still within the range of hopping transport model (below the critical value of $10^{22} \mathrm{~cm}^{-3}$ ).

\section{References}

1. Ovshinsky, S. R. Reversible electrical switching phenomena in disordered structures. Phys. Rev. Lett. 21, 1450-1453 (1968).

2. Waser, R. \& Aono, M. Nanoionics-based resistive switching memories. Nat. Mater. 6, 833-840 (2007).

3. Yang, J. J., Strukov, D. B. \& Stewart, D. R. Memristive devices for computing. Nat. Nanotechnol. 8, 13-24 (2013).

4. Borghetti, J. et al. 'Memristive' switches enable 'stateful' logic operations via material implication. Nature 464, 873-876 (2010).

5. Pershin, Y. V. \& Ventra, M. D. Practical approach to programmable analog circuits with memristors. IEEE Trans. Circuits Syst. I-Reg. Papers 57, 1857-1864 (2010).

6. Lee, M.-J. et al. A fast, high-endurance and scalable non-volatile memory device made from asymmetric $\mathrm{Ta}_{2} \mathrm{O}_{5-\mathrm{x}} / \mathrm{TaO}_{2-\mathrm{x}}$ bilayer structures. Nat. Mater. 10, 625-630 (2011).

7. Kwon, D.-H. et al. Atomic structure of conducting nanofilaments in $\mathrm{TiO}_{2}$ resistive switching memory. Nat. Nanotechnol. 5, 148-153 (2010).

8. Liu, Q. et al. Real-time observation on dynamic growth/dissolution of conductive filaments in oxide-electrolyte-based ReRAM. Adv. Mater. 24, 1844-1849 (2012).

9. Miao, F. et al. Anatomy of a nanoscale conduction channel reveals the mechanism of a high-performance memristor. Adv. Mater. 23, 5633-5640 (2011).

10. Yu, S., Guan, X. \& Wong, H. S. P. Conduction mechanism of TiN/HfO $/ \mathrm{Pt}$ resistive switching memory: A trap-assisted-tunneling model. Appl. Phys. Lett. 99, 063507 (2011).

11. Xu, N. et al. A unified physical model of switching behavior in oxide-based RRAM. Symp. VLSI Tech. 100-101 (2008).

12. Tseng, H. -C. et al. Investigating the improvement of resistive switching trends after post-forming negative bias stress treatment. Appl. Phys. Lett. 99, 132104 (2011).

13. Syu, Y. -E. et al. Atomic-level quantized reaction of $\mathrm{HfO}_{\mathrm{x}}$ memristor. Appl. Phys. Lett. 102, 172903 (2013).

14. Rowe, D. M. et al. CRC Handbook of Thermoelectrics (CRC, Boca Raton, 1995).

15. Cho, S. et al. Thermoelectric imaging of structural disorder in epitaxial graphene. Nat. Mater. 12, 913-918 (2013).

16. Walter, M. et al. Seebeck effect in magnetic tunnel junctions. Nat. Mater. 10, 742-746 (2011).

17. Fritzsche, H. A general expression for the thermoelectric power. Solid State Commun. 9, 1813-1815 (1971).

18. Pernstich, K. P., Rossner, B. \& Batlogg, B. Field-effect-modulated Seebeck coefficient in organic semiconductors. Nat. Mater. 7, 321-325 (2008).
19. Germs, W. C., Guo, K., Janssen, R. A. J. \& Kemerink, M. Unusual thermoelectric behavior indicating a hopping to bandlike transport transition in pentacene. Phys. Rev. Lett. 109, 016601 (2012).

20. Cai, J. \& Mahan, G. D. Effective Seebeck coefficient for semiconductors. Phys Rev. B 74, 075201 (2006).

21. Cao, J., Fan, W., Zheng, H. \& Wu, J. Thermoelectric effect across the metalinsulator domain walls in $\mathrm{VO}_{2}$ microbeams. Nano Lett. 9, 4001-4006 (2009).

22. Nguyen, T. H. \& O'Leary, S. K. The dependence of the Fermi level on temperature, doping concentration, and disorder in disordered semiconductors. J. Appl. Phys. 88, 3479 (2000).

23. Emin, D. Thermoelectric power due to electronic hopping motion. Phys. Rev. Lett. 35, 882-885 (1975).

24. Wu, P. M. et al. Thermoelectric characterization of electronic properties of GaMnAs nanowires. J. Nanotechnol. 2012, 1-5 (2012).

25. Navasery, M. et al. Characterization and conduction mechanism of $\mathrm{La}_{5 / 8} \mathrm{Sr}_{3 / 8} \mathrm{MnO}_{3}$ thin films prepared by pulsed laser deposition on different substrates. Int. J. Electrochem. Sci. 8, 6905-6921 (2013).

26. Mahendiran, R. et al. Structure, electron-transport properties, and giant magnetoresistance of hole-doped $\mathrm{LaMnO}_{3}$ systems. Phys. Rev. B 53, 3348-3358 (1996).

27. Banerjee, A., Pal, S. \& Chaudhuri, B. K. Nature of small-polaron hopping conduction and the effect of $\mathrm{Cr}$ doping on the transport properties of rare-earth manganite $\mathrm{La}_{0.5} \mathrm{~Pb}_{0.5} \mathrm{Mn}_{1-\mathrm{x}} \mathrm{Cr}_{\mathrm{x}} \mathrm{O}_{3}$. J. Chem. Phys. 115, 1550-1158 (2001).

28. Austen, I. G. \& Mott, N. F. Polarons in crystalline and non-crystalline materials Adv. Phys. 18, 41-102 (1969).

29. Hildebrandt, E., Kurian, J. \& Alff, L. Physical properties and band structure of reactive molecular beam epitaxy grown oxygen engineered $\mathrm{HfO}_{2 \pm \mathrm{x}}$. J. Appl. Phys. 112, 114112 (2012).

30. Panzer, M. A. et al. Thermal properties of ultrathin hafnium oxide gate dielectric films. IEEE Electron Device Lett. 30, 1269-1271 (2009).

31. Lee, S. M. \& Gahill, D. G. Thermal conductivity of sputtered oxide films. Phys. Rev. B 52, 253-257 (1995).

32. Miller, A. \& Abrahams, E. Impurity conduction at low concentrations. Phys. Rev. 120, 745-755 (1960).

33. Broqvist, P. \& Pasquarello, A. Amorphous hafnium silicates: structural, electronic and dielectric properties. Microelectron. Eng. 84, 2416-2419 (2007)

\section{Acknowledgements}

We thank Professor C.S. Hwang from Seoul National University for valuable discussions. This work was supported by the Ministry of Science and Technology of China under grant numbers 2011CBA00602, 2010CB934200, 2011AA010401, 014AA032900, 2013CBA01604 and 2011AA010402, and NSFC under grant numbers 61221004, $61334007,61322408,61274091,61106119$ and 61106082

\section{Author contributions}

M.W., C.B., L.L. and M.L. designed this work; M.W.and C.B. designed and fabricated the devices, and carried out the Seebeck effect measurement; M.W. preformed the temperature-dependent resistance measurement; L.L. interpreted the experiment results; all authors discussed the experiments and contributed to the manuscript preparation. M.L. coordinated and supervised the whole work.

\section{Additional information}

Supplementary Information accompanies this paper at http://www.nature.com/ naturecommunications

Competing financial interests: The authors declare no competing financial interests.

Reprints and permission information is available online at http://npg.nature.com/ reprintsandpermissions/

How to cite this article: Wang, M. et al. Thermoelectric Seebeck effect in oxide-based resistive switching memory. Nat. Commun. 5:4598 doi: 10.1038/ncomms5598 (2014).

This work is licensed under a Creative Commons Attribution 4.0 International License. The images or other third party material in this article are included in the article's Creative Commons license, unless indicated otherwise in the credit line; if the material is not included under the Creative Commons license, users will need to obtain permission from the license holder to reproduce the material. To view a copy of this license, visit http://creativecommons.org/licenses/by/4.0/ 